\title{
Orthodontic retainers: Analysis of prescriptions sent to laboratories
}

Washington Komatsu Assumpção', George Kenji Bezerra Ota', Rívea Inês Ferreira², Flávio Augusto Cotrim-Ferreira²

Objective: To investigate the most commonly fabricated orthodontic retainers.

Methods: Information on the type and amount of maxillary and mandibular retainers produced in a three-month period was collected from six laboratories in the cities of São Paulo, Mauá and Guarulhos - Brazil. The retainers were grouped according to the total production. For the maxillary arch, the groups were: $1 \mathrm{~S}$ - Begg retainer, $2 \mathrm{~S}$ Hawley retainer, $3 \mathrm{~S}$ - transpalatal arch retainer, $4 \mathrm{~S}$ - buccal resin-arch retainer and $5 \mathrm{~S}$ - vacuum-formed retainer, Planas appliance, bonded lingual retainer and V-loop bonded lingual retainer. The groups relative to the mandibular arch were: 1I - 3-3 bonded lingual retainer (canine to canine), 2I - Hawley retainer and V-loop bonded lingual retainer, 3I - Begg retainer, 4I - buccal resin-arch retainer, vacuum-formed retainer and Planas appliance. The data were presented in box plots. Groups were compared using the Student's-t test with Bonferroni correction.

Results: The average of maxillary appliances fabricated ranged from 189.5 (1S) to 3.95 (5S). There were significant differences between groups $1 S$ versus $5 \mathrm{~S}$ and $2 \mathrm{~S}$ versus $5 \mathrm{~S}(\mathrm{p}<0.0001)$. Mean values for the mandibular retainers ranged from 55.3 (1I) to 4.2 (4I). Significant difference was observed between groups 2 I and 4 I ( $<<0.0001)$.

Conclusions: For the maxillary arch, the most requested retainers were Begg and Hawley retainers. Regarding the mandibular arch, bonded lingual retainers and Hawley retainer were the most frequent ones.

Keywords: Orthodontics. Corrective. Orthodontic appliances. Relapse.

${ }^{1}$ Specialist in Orthodontics, School of Dentistry, University of the City of São Paulo (UNICID).

${ }^{2}$ Associate Professor, MSc Program in Orthodontics, UNICID.
How to cite this article: Assumpção WK, Ota GKB, Ferreira RI, Cotrim-Ferreira FA. Orthodontic retainers: Analysis of prescriptions sent to laboratories. Dental Press J Orthod. 2012 Mar-Apr;17(2):36.el-6.

\footnotetext{
Submitted: October 30, 2008 - Revised and accepted: March 2, 2009

» The authors report no commercial, proprietary, or financial interest in the products or companies described in this article.

Contact address: Rívea Inês Ferreira

Rua Cesário Galeno, 448 Bloco A - CEP: 03071-000 - Tatuapé / SP - Brazil

E-mail: riveaines@yahoo.com
} 


\section{INTRODUCTION}

The relapse of orthodontic treatment may be associated to several aspects, including periodontal and occlusal factors, pressure from the oral soft tissues and growth. ${ }^{14}$ Therefore, orthodontic retainers have the substantial function of providing stability for the outcomes achieved at the end of treatment., ${ }^{3,7,8,13,18}$

The orthodontic retainers may be removable, fixed, active or passive., ${ }^{4,5}$ These appliances should maintain the six keys to normal occlusion of Andrews (curve of Spee, molar relationship, adequate crown angulation and inclination, absence of rotations and diastema), achieved by orthodontic treatment, as well as periodontal health, ${ }^{4,6,18,22}$ absence of harmful habits, muscular balance and the established functional occlusion. ${ }^{13}$

The bonded lingual retainer fixed on the surfaces of mandibular anterior teeth is considered an effective method for long-term stabilization ${ }^{6,17}$ and is well accepted by the patients because it is esthetic and does not depend on their compliance. A clinical trial comparing fixed retainers manufactured with multistranded metallic wire and a direct-bonded polyethylene ribbon-reinforced resin composite, from mandibular canine to canine, revealed that the polyethylene strip had a mean durability of 11.5 months, compared to 23.6 months for the metallic wire retention. ${ }^{19}$ According to another study, $74 \%$ of fixed mandibular 3-3 lingual retainers were still present in the patients' mouth after four years and only $2 \%$ of patients exhibited gingival alterations after five years of use. ${ }^{10}$

Regarding the removable retainers, the Hawley retainer and its variations are the most indicated. ${ }^{1}$ In cases of orthodontic treatment with four premolar extraction followed by use of Hawley retainers, both in maxillary and mandibular arches, there was a significant increase in occlusal contacts of posterior teeth. ${ }^{20}$ There is some evidence that these retainers are more hygienic than other appliances..$^{18}$ However, patient compliance is a matter of concern when removable appliances are indicated.

A study conducted in the United Kingdom evaluated the cost-benefit relationship and patient satisfaction with the use of Hawley retainers and vacuumformed retainers. ${ }^{9}$ The results indicated that the vacuum-formed retainers presented better cost-benefit relationship. The first retainers had lower costs for fabrication and repair, caused less discomfort to the patients and did not impair the speech, which confirmed its preference at this geographic region. ${ }^{9}$

Several devices are used for orthodontic retention and the frequency of indication of some appliances is still controversial, ${ }^{2}$ since it may vary depending on the geographic region and patient characteristics. Thus, the aim of this study was to assess the most frequently requested orthodontic retainers to laboratories located in São Paulo and two neighbor cities. This survey indirectly aimed at demonstrating the preferences of part of orthodontists from the metropolitan area of São Paulo, and also may contribute to investigations on orthodontic retention, especially concerning the indications of the available retainers.

\section{MATERIAL AND METHODS}

This study was conducted in accordance with the ethical principles foreseen in the Resolution 196/96 of the Brazilian National Health Council and was approved by the Institutional Review Board.

Information on the type and amount of retainers for the maxillary and mandibular dental arches, fabricated in a continuous period of three months in six laboratories that deliver these services at the cities of São Paulo ( $=4)$, Mauá ( $\mathrm{n}=1)$ and Guarulhos $(\mathrm{n}=1)$, was collected. After consenting to the voluntary participation in this study, the directors of the laboratories were instructed to fill monthly spreadsheets in which they recorded data on the retainers requested by orthodontists. Seven laboratories were initially included in the study, yet data from one of them were excluded because of incompleteness.

The descriptive analysis comprised evaluation of numbers related to the production of each retainer, formation of clusters/groups and graphic presentation in box plots.

The cluster analysis was used to group the retainers according to the total amount of appliances fabricated in the study period. This kind of analysis is applied to group collections of data based on the similarity between them, which in the present study corresponded to the numbers related to the total production, and not to the physical aspect or method of utilization. The clusters are determined to achieve a high degree of homogeneity within groups and an increased degree of heterogeneity between them. 
The formation of clusters was influenced by the amount of retainers produced. Hence, retainers presenting the greatest production were gathered in the same group, in descending order of fabrication.

For the maxillary arch, in decreasing order of demand, the groups were: $1 \mathrm{~S}$ - Begg retainer, $2 \mathrm{~S}$ - Hawley retainer, $3 \mathrm{~S}$ - transpalatal arch retainer, $4 \mathrm{~S}$ - buccal resin-arch retainer, and $5 \mathrm{~S}$ - vacuum-formed retainer using acetate or polyethylene, Planas appliance, bonded lingual retainer and V-loop bonded lingual retainer or hygienic retainer. The groups for the mandibular arch were: 1I - 3-3 bonded lingual retainer, 2I - Hawley retainer and V-loop bonded lingual retainer or hygienic retainer, 3I - Begg retainer, and 4I - buccal resin-arch retainer, vacuum-formed retainer using acetate or polyethylene and Planas appliance.

The groups of maxillary and mandibular retainers were compared using the Student's-t test $(\alpha=5 \%)$ with Bonferroni correction. It is important to explain that, since the sample was small, did not present normal distribution and the total production of some retainers corresponded to zero, data were converted into ranks, which allowed the use of the above mentioned parametric test.

\section{RESULTS}

The distribution of maxillary retainers fabricated by the six laboratories surveyed, in absolute numbers, is shown in Figure 1. The production of Begg and Hawley retainers was much higher compared to the other appliances. The data from Figure 2 and Table 1 corroborate the observation related to Figure 1.

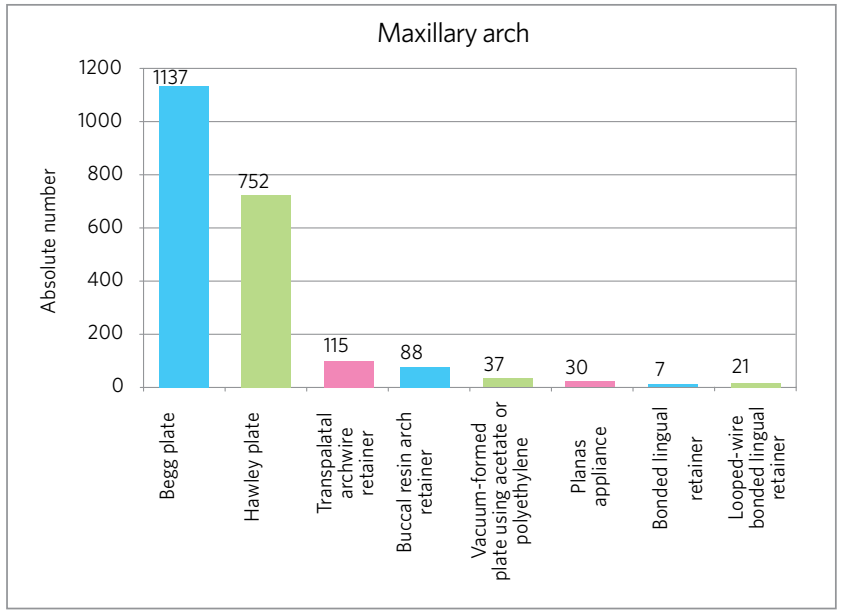

Figure 1- Absolute numbers corresponding to the total production of maxillary orthodontic retainers in six laboratories.
The most frequently fabricated retainer was the Begg retainer (1S), however, this appliance also presented the greatest variation. The standard deviation was much higher than the mean for this retainer (Table 1). The second maxillary retainer most often requested was the Hawley retainer (2S). The other groups had progressively lower mean frequencies of fabrication, with significantly lower mean value for the group $5 \mathrm{~S}$ compared to groups $1 \mathrm{~S}$ and $2 \mathrm{~S}$ (Table 2).

In absolute numbers, the production of mandibular retainers was much lower compared to the maxillary appliances (Figs 1 and 3). The most often fabricated retainer was the 3-3 bonded lingual retainer. Concerning the magnitude of production, the maxillary Begg retainer reached an absolute number of 1,137 in the three months surveyed. On the other hand, 332 were the amount of 3-3 bonded lingual retainers produced in the same period.

Table 1 - Measures of central tendency (median and mean) and variability (standard deviation, minimum and maximum values) relative to the groups of maxillary orthodontic retainers.

\begin{tabular}{ccccccc}
\hline Group & $\mathbf{n}$ & Median & Mean & s.d. & Minimum & Maximum \\
\hline 1S & 6 & 134.0 & 189.5 & 215.2 & 23.0 & 592.0 \\
2S & 6 & 116.0 & 125.3 & 52.0 & 45.0 & 190.0 \\
3S & 6 & 11.0 & 19.2 & 19.8 & 0.0 & 52.0 \\
4S & 6 & 10.0 & 14.7 & 18.8 & 0.0 & 47.0 \\
5S & 24 & 0.0 & 3.95 & 8.3 & 0.0 & 30.0 \\
\hline
\end{tabular}

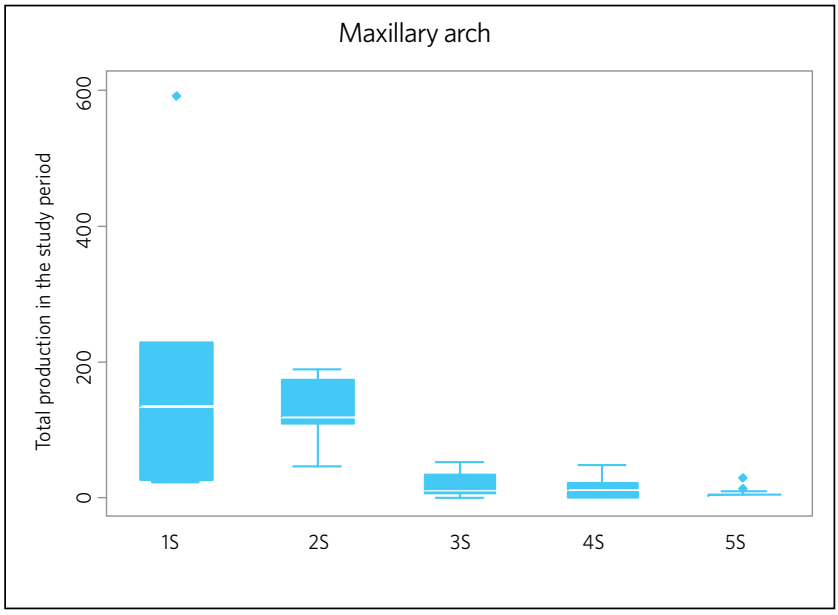

Figure 2 - Box plots representing the distribution pattern for data relative to the production of maxillary orthodontic retainers. 
According to Figure 4 and Table 3, the most frequently fabricated mandibular retainer was the 3-3 bonded lingual retainer (1I), but it also demonstrated the highest standard deviation. This retainer was followed by the appliances in group 2I (Hawley retainer and V-loop bonded lingual retainer). Based on the comparisons shown in Table 4, the retainers in group 2I were fabricated with significantly higher

Table 2 - Comparison between groups of maxillary retainers.

\begin{tabular}{ccc} 
Comparisons & $\mathbf{t}$ & p-value \\
\hline 1S vs 2S & -0.31 & 0.7602 \\
$1 S$ vs 3S & 2.36 & 0.0479 \\
1S vs 4S & 3.05 & 0.0202 \\
1S vs 5S & 8.35 & 0.0000 \\
2S vs 3S & 2.77 & 0.0357 \\
2S vs 4S & 3.37 & 0.0183 \\
2S vs 5S & 11.74 & 0.0000 \\
3S vs 4S & 1.01 & 0.3358 \\
3S vs 5S & 2.88 & 0.0222 \\
4S vs 5S & 1.04 & 0.3224 \\
\hline
\end{tabular}

Bonferroni's correction: $p<0.005$.

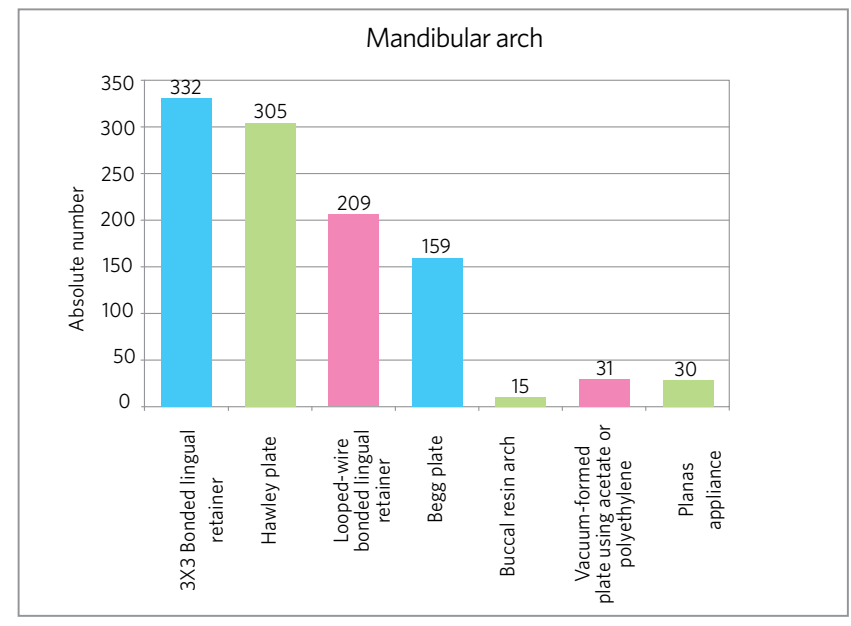

Figure 3 - Absolute numbers corresponding to the total production of mandibular orthodontic retainers in six laboratories.

Table 3 - Measures of central tendency (median and mean) and variability (standard deviation, minimum and maximum values) relative to the groups of mandibular orthodontic retainers.

\begin{tabular}{ccccccc}
\hline Groups & $\mathbf{n}$ & Median & Mean & s.d. & Minimum & Maximum \\
\hline 11 & 6 & 9.5 & 55.3 & 78.0 & 0.0 & 163.0 \\
21 & 12 & 20.5 & 42.8 & 41.7 & 3.0 & 115.0 \\
31 & 6 & 4.5 & 26.5 & 38.2 & 0.0 & 85.0 \\
41 & 18 & 0.0 & 4.2 & 8.8 & 0.0 & 30.0 \\
\hline
\end{tabular}

frequencies compared to group 4I (buccal resin-arch retainer, vacuum-formed retainer using acetate or polyethylene and Planas appliance).

\section{DISCUSSION}

The orthodontic literature shows wide discussions on retainers, specifically concerning their indication, type of appliance and time during which they should be maintained after completion of active orthodontic treatment. While some authors indicate the use of retainers for an indefinite period in some situations, ${ }^{14}$ others advocate that the retention period should be as long as the active treatment lasted. ${ }^{3}$

The use of maxillary retainers is necessary mainly due to the possibility of relapse of diastemas and rotations, as well as for maintenance of intercanine and intermolar widths after maxillary expansion..$^{3,5,8,13,22}$ According to this study findings, the Begg and Hawley retainers were the maxillary retainers most often requested by orthodontists to the laboratories surveyed, with mean productions of 189.5 and 125.3, respectively, in a three-month period. The production

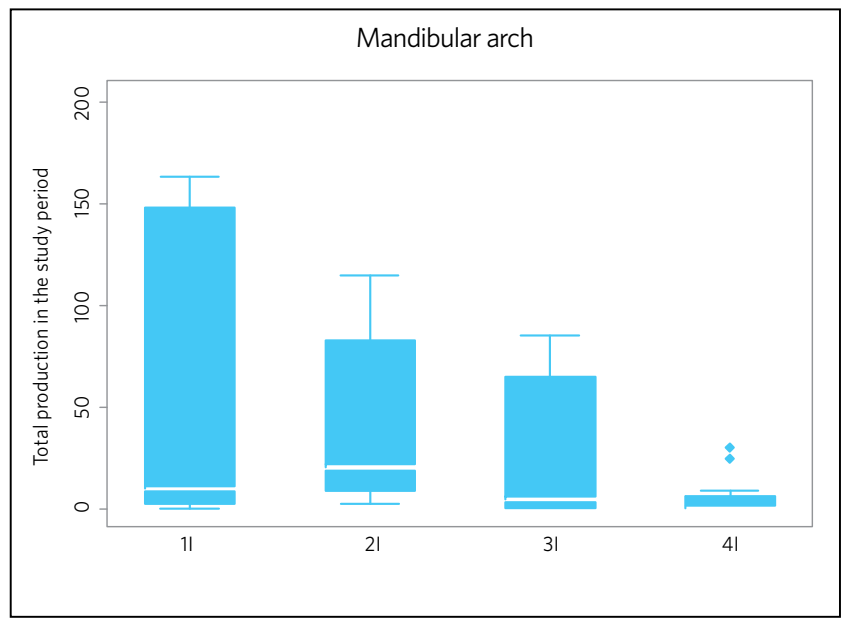

Figure 4- Box plots representing the distribution pattern for data relative to the production of mandibular orthodontic retainers.

Table 4 - Comparison between groups of mandibular retainers.

\begin{tabular}{|ccc|}
\hline Comparisons & $\mathbf{t}$ & p-value \\
\hline 1I vs 2I & -0.90 & 0.4031 \\
1I vs 3I & 0.63 & 0.5381 \\
1I vs 4I & 2.52 & 0.0367 \\
2I vs 3I & 1.68 & 0.1454 \\
2I vs 4I & 6.58 & 0.0000 \\
3I vs 4I & 1.59 & 0.1536 \\
\hline
\end{tabular}

*Bonferroni's correction: $p$-value $<0.0083$ 
of these two types of retainers was significantly higher compared to the others in group 5S (Table 2). These data agree with other studies that indicate the Hawley retainer and its variations as the most used removable appliances for maxillary retention..$^{18,22}$

The Hawley retainer is fabricated with acrylic resin, covering the palate, and presents a stainless steel buccal arch that usually extends from the distal surfaces of canines, contouring the buccal aspect of maxillary anterior teeth. Moreover, this appliance has additional retentions as Adams or circumferential clasps. Conversely, in the Hawley retainer with continuous arch or Begg retainer, the buccal arch is initiated on the distal surfaces of second molars and contours the buccal aspects of anterior and posterior teeth, ${ }^{18}$ without the need of retention clasps. However, in some cases, clasps may be added to stabilize the continuous arch.

The Hawley retainer has high durability, ${ }^{18}$ provides better intercuspation of posterior teeth ${ }^{20}$ and may promote small tooth movements. ${ }^{3}$ Some authors state that the Hawley retainer may last up to 15 years, allows easy hygiene maintenance, has relatively low cost and does not cause discomfort to the patient during utilization. ${ }^{1,18}$ Nevertheless, the intercuspation maybe impaired in areas with occlusal interferences from the stainless steel wire of the retention clasps. The modification from the Hawley to the Begg retainer would provide a solution to minimize the problem of occlusal interferences, by eliminating the retention clasps. ${ }^{18}$

Retention in the mandibular arch is even more important, not only to prevent relapses, but also to avoid the secondary crowding of incisors, ${ }^{16,17}$ which may be explained as an anatomical-physiological adaptation phenomenon. ${ }^{7,8,13}$ As demonstrated in Figure 3 and Table 3, the mandibular retainers most frequently requested were the 3-3 bonded lingual retainer (from canine to canine), the Hawley retainer and the V-loop bonded lingual retainer or hygienic retainer. Considering that the two latter appliances were grouped into a cluster (2I), the mean values relative to their production in the study period were 55.3 (II) and 42.8 (2I), respectively. However, the detailed analysis of Table 3 reveals great discrepancy between the median (9.5) and mean (55.3) calculated for the canineto-canine bonded lingual retainer. The median value calls attention to the fact that $50 \%$ of the laboratories produced up to 9 appliances. After observation of the crude data, it was found that very high numbers of this appliance were fabricated in a single laboratory, which increased the mean value. Thus, because of the lack of consistency in the distribution of data on the canine-to-canine bonded lingual retainer, the Hawley retainer and the V-loop bonded lingual retainer or hygienic retainer were significantly more requested than the other appliances in group 4I (Table 4). It should be highlighted that data in this study were based on the outsourced production of orthodontic retainers, and consequently information on the type of appliance most often used in the mandibular arch may be masked. This is explained by the fact that the bonded lingual retainer may be easily manufactured in the dental office and, therefore, many orthodontists do not request this type of appliance to laboratories.

The canine-to-canine bonded mandibular lingual retainer was introduced in the United States in the 1970s and has been used as an integral part of orthodontic treatment since then. ${ }^{11,16,23}$ Most mandibular retainers are bonded/fixed, because mandibular removable plates have a large bulk of acrylic on the lingual aspect, decreasing the tongue space, impairing the speech and swallowing., ${ }^{7,22}$ This drawback would preclude adequate utilization of the appliance, damaging the treatment outcomes. Notably, the greatest disadvantage of removable retainers is the need of patient compliance, both in maxillary and mandibular arches. ${ }^{1,2,4,15,18,20,22}$

The use of mandibular fixed retainers fabricated with smooth stainless steel wire bonded only to the canines is encouraged. ${ }^{17}$ The greatest advantage of canine-to-canine bonded mandibular retainers compared to removable retainers lies in the esthetic quality, because they are not easily perceptible and are well tolerated by patients. ${ }^{16,24}$ This type of mandibular retainer is also adequate in cases in which the periodontal support is reduced. ${ }^{18}$

The difficulty of patients to perform the hygiene of proximal surfaces in regions where the fixed retainer is bonded led to its modification, in order to facilitate the use of dental floss within the interproximal spaces. For that purpose, loops may be fabricated in the stainless steel archwire in opposite direction than the gingival papillae. However, a comparative study analyzing the fixed retainers modified with loops versus 
without loops and bonded only to the mandibular canines evidenced higher accumulation of biofilm and dental calculus at the region of the modified fixed retainer, with gingival inflammation probably caused by the greater length of wire and presence of angles in the loops. ${ }^{21}$ Therefore, these loops would constitute the most retentive areas, which would impair the hygiene.

A study on the effectiveness of bonded retainers and their effects on the periodontal status, conducted for at least 20 years follow-up, reported that these appliances are very effective to maintain the tooth alignment and allow the maintenance of acceptable levels of hygiene and periodontal health. ${ }^{6}$ The authors further mentioned that when good bonding techniques are used for the placement of fixed retainers they are not frequently loosened by breakage, thus not contraindicating their prescription. ${ }^{6}$

The duration of retainer wear is related to the patient age, characteristics and severity of malocclusion.
The oral habits and other etiologic factors of malocclusions, as well as the orthodontic mechanics employed and the clinical experience of the orthodontist are also relevant factors to estimate the retention period. ${ }^{14,21}$ From a clinical standpoint, maintenance of the mandibular retainer for an undetermined period assures the outcomes of active orthodontic treatment, since it is not possible to predict the cases that will present relapse., ${ }^{5,10,17,18}$ The stability should be monitored and controlled by the orthodontists during the routine assessments of retainers for a long time. ${ }^{12}$

\section{CONCLUSIONS}

1. For the maxillary arch, the Begg and Hawley retainers were the orthodontic retainers most often requested to the laboratories surveyed.

2. Among the mandibular retainers, the most frequently requested were the bonded lingual retainers and Hawley retainer.

\section{REFERENCES}

1. Al-Suliaman S, Hashim HA, Cordovez JL. The reinforced removable retainer. J Contemp Dent Pract. 2006;7(2):145-52.

2. Atack N, Harradine N, Sandy JR, Ireland AJ. Which way forward? Fixed or removable lower retainers. Angle Orthod. 2007;77(6):954-9.

3. Bengtson AL, Bengtson NG. Contenção ortodôntica: sugestão para uma placa retentora. Rev Assoc Paul Cir Dent. 1996;50(4):333-6.

4. Bicalho JS, Bicalho KT. Descrição do método de contenção fixa, com livre acesso do fio dental. Rev Dental Press Ortod Ortop Facial. 2001;6(5):97-104.

5. Bicalho JS, Bicalho KT. Descrição do método de contenção fixa, com livre acesso do fio dental. Rev Clin Ortod Dental Press. 2002;1(1):9-13.

6. Booth FA, Edelman JM, Proffit WR. Twenty-year follow-up of patients with permanently bonded mandibular canine-to-canine retainers. Am J Orthod Dentofacial Orthop. 2008;133(1):70-6.

7. Cerny R. The reliability of bonded lingual retainers. Aust Orthod J. 2007;23(1):1-6

8. Fonte PP, Fonte MLM. Contenção ortodôntica. Rev Fac Odontol Pernambuco. 2000;18(1-3):9-23

9. Hichens L, Rowland H, Williams A, Hollinghurst S, Ewings P, Clark S, et al. Costeffectiveness and patient satisfaction: Hawley and vacuum-formed retainers. Eur J Orthod. 2007;29(4):372-8

10. Johnsson AC, Tofeldt LN, Kjellberg H. Subjective evaluation of orthodontic treatment and potential side effects of bonded lingual retainers. Swed Dent J. 2007;31(1):35-44.

11. Katsaros C, Livas C, Renkema AM. Unexpected complications of bonded mandibular lingual retainers. Am J Orthod Dentofacial Orthop. 2007;132(6):838-41

12. Kortam SI, El-Beialy AR, Mostafa YA. Retention checklist. J Clin Orthod. 2007;41(7):382-3.

13. Kuramae M, Almeida MHC, Noüer DF, Magnani MBBA. Principais fatores relacionados à estabilidade ortodôntica: uma revisão de literatura. J Bras Ortodon Ortop Facial. 2002;7(39):194-200.
14. Littlewood SJ, Millett DT, Doubleday B, Bearn DR, Worthington HV. Retention procedures for stabilising tooth position after treatment with orthodontic braces. Cochrane Database Syst Rev. 200625;(1):CD002283.

15. Lumsden KW, Saidler G, Mccoll JH. Breakage incidence with direct bonded lingual retainers. Br J Orthod. 1999;26(3):191-4.

16. Pandis N, Vlahopoulos K, Madianos ETP. Long-term periodontal status of patients with mandibular lingual fixed retention. Eur J Orthod. 2007;29(5):471-6.

17. Renkema AM, Al-Assad S, Bronkhorst E, Weindel S, Katsaros C, Lisson JA Effectiveness of lingual retainers bonded to the canines in preventing mandibular incisor relapse. Am J Orthod Dentofacial Orthop. 2008;134(2):179e1-8.

18. Rinchuse DJ, Miles PG, Sheridan JJ. Orthodontic retention and stability: a clinical perspective. J Clin Orthod. 2007;41(3):125-32.

19. Rose $\mathrm{E}$, Jonas E. Clinical comparison of a multistranded wire and a direct-bonded polyethylene ribbon-reinforced resin composite used for lingual retention. Quintessence Int. 2002;33(8):579-83.

20. Sauget E, Covell DA Jr, Boero RP, Lieber WS. Comparison of occlusal contacts with use of Hawley and clear overlay retainers. Angle Orthod. 1997;67(3):22330.

21. Shirasu BK, Hayacibara RM, Ramos AL. Comparação de parâmetros periodontais após utilização de contenção convencional $3 \times 3$ plana e contenção modificada. Rev Dental Press Ortod Ortop Facial. 2007;12(1):41-7.

22. Silveira M. O uso do P.E.T. (Polietileno Tereftalato) na construção de contenções ortodônticas removíveis. J Bras Ortodon Ortop Facial. 1998;3(14):33-7.

23. Upadhyay M, Yadav S, Keluskar KM. Molar bands for precision bonding of lingual retainers. J Orthod. 2007;34(1):12-5.

24. Zachrisson BU. Differential retention with bonded retainers. World J Orthod. 2007:8(2):190-6. 